\title{
Les filières de déchets de très faible activité à EDF
}

\author{
Rémy HILMOINE*, Louis-Philippe CASSEAU**
}

(Manuscrit reçu le 18 février 1998, révisé le 25 août 1998, accepté le 18 mai 1999)

RÉSUMÉ Après avoir rappelé les enjeux liés à la future gestion des déchets de très faible activité pour EDF en terme de tonnages, on décrit à quoi conduit l'application des principes liés à cette gestion : zonage des installations, mise en place de filières, traçabilité des déchets et contrôles associés. L'origine des déchets est ensuite décrite de manière qualitative et quantitative, notamment l'étalement de leur production dans le temps. La gestion des TFA envisagée à EDF est abordée : stockage dans un centre de stockage dédié et filières de traitement/élimination dont l'impact radiologique et le coût seraient jugés acceptables. L'exemple d'étude de filières de traitement des huiles TFA est alors développé : notamment les hypothèses retenues et les principaux résultats en matière d'impact radiologique. On justifie le choix de l'incinération et l'on décrit les difficultés pressenties pour sa mise en ceuvre industrielle. Les autres études en cours sont citées et les principales conclusions sont tirées : période charnière, pour le sujet, entre réflexion et action ; adaptation des démantèlements engagés aux principes en cours d'émergence et forte communication associée.

ABSTRACT Lowlevel wastes pathways at EDF.

First, what are, for EDF, the main issues dealing with the future management of low level wastes (LLW) will be recalled; and followed by a description of what are the implications of implementing these management principles: areas zoning, set up of pathways, traceability of the wastes and associated controls. The origin of the wastes will then be described using both qualitative and quantitative approaches; the description will specifically address the spreading of wastes production in time. LLW management at EDF will then be envisaged: storage in a specific discharge, pathways for treatment and elimination of wastes with acceptable radiological impact and costs. The example of LLW oils will be developed: particularly as far as hypothesis and results concerning the radiological impacts are concerned. The choice of incineration will then be justified, however expected difficulties to implement it industrially will be pointed out. Other on going studies and their main results will be mentioned: the present time is a turning point on that issue between thought and action; the on going dismantling must take into account the emerging principles and give rise to good communication.

\footnotetext{
* Électricité de France, Pôle Industrie/DPN/DSRE, Site Cap Ampère, 1 place Pleyel, 93282 Saint-Denis cedex, France

*** EDF-EPN/DSRE, 1 place Pleyel, bât. tête Pleyel B, 93282 Saint-Denis cedex, France
} 


\section{Généralités}

La dénomination « déchets de très faible activité » (TFA dans la suite du texte), s'applique aux déchets issus de l'exploitation, mais surtout du démantèlement des installations nucléaires de base :

- dès l'instant qu'ils appartiennent à une partie de l'installation considérée comme zone pouvant engendrer des déchets à radioactivité artificielle ajoutée (Zone Déchets Nucléaire),

- dont le niveau d'activité est inférieur à celui des déchets « faible et moyenne activité ». Pour les TFA, l'activité massique moyenne est inférieure à $10 \mathrm{~Bq} / \mathrm{g}$, avec des valeurs maximales inférieures à $100 \mathrm{~Bq} / \mathrm{g}$.

On estime à environ 11 millions de tonnes la quantité de déchets qui sera produite par les 65 tranches du parc REP + UNGG, dont environ les trois quarts correspondent au niveau 3 des démantèlements, un quart au niveau 2 et le reste, moins de $1 \%$, est produit en cours d'exploitation ( $c f$. tab. I).

\section{TABLEAU I}

Les déchets des réacteurs EDF (en tonnes). - Estimation 1997. Reactors wastes (in tons).

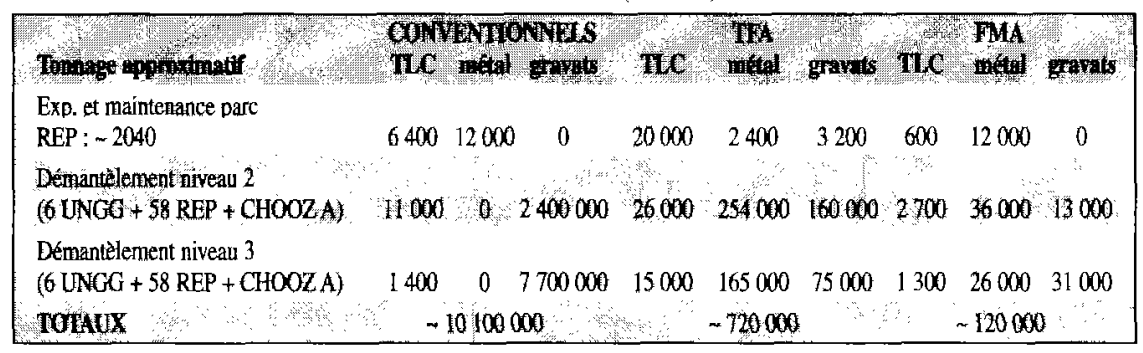

TLC : déchets technologiques + liquides combustibles + calorifuges et câbles électriques.

Cependant, sur cette quantité importante, seulement moins de 1 million de tonnes répondront finalement, au sens des filières d'évacuation, à la dénomination «TFA » avec une activité massique moyenne comprise entre une valeur se rapprochant de la radioactivité naturelle (environ $0,1 \mathrm{~Bq} / \mathrm{g}$ ) et la valeur maximale de $100 \mathrm{~Bq} / \mathrm{g}$ évoquée ci-dessus. L'autre partie ( 10 millions de tonnes, des bétons de génie civil essentiellement) est considérée comme «conventionnelle». Lors des démantèlements, il convient de garantir le non-mélange de ces deux catégories de déchets.

\subsection{Les principes de gestion}

La garantie de non-mélange et de traitement approprié pour les catégories de déchets évoquées ci-dessus repose sur des principes de gestion analogues à ceux retenus pour 
des déchets conventionnels ou en cours d'élaboration. Ils sont rappelés brièvement ci-après :

Responsabilité de l'exploitant: l'exploitant, producteur des déchets, en demeure responsable même quand ils ont quitté les lieux de production, soit en vue de leur stockage en décharge, soit pour être éliminés ou recyclés.

Contrôle de l'administration : l'administration ne dispose pas encore d'une réglementation lui permettant d'effectuer comme elle l'entend des contrôles chez le producteur et tout au long de la chaîne. Fruit d'une concertation entre les principaux producteurs (EDF, CEA, COGEMA) et les ministères concernés (Industrie, Environnement, Santé), une réglementation concernant la gestion des déchets TFA est en cours d'émergence. Elle prévoit :

a) une extension du décret de 1966 relatif à la radioprotection vis-à-vis des matières dont l'activité massique moyenne est inférieure à $100 \mathrm{~Bq} / \mathrm{g}$;

b) la mise en place, en application d'une circulaire, d'études déchets pour chaque installation nucléaire (INB ou ICPE) $\left({ }^{*}\right)$, avec des principes analogues à ceux retenus pour les études déchets réalisées pour les installations industrielles classiques. Elles devront justifier pour chaque déchet inventorié, les filières de traitement/élimination retenues ainsi que les spécifications techniques et procédures associées (traçabilité, ...).

Une telle étude, réalisée à titre expérimental en 1995 pour le site du BLAYAIS, a été engagée sur tous les CNPE (**) au cours de l'année 1998, selon un canevas établi à la fin de 1997 ;

c) la création d'un nouveau type d'ICPE permettant le stockage de déchets TFA;

d) un contrôle de l'Administration sur les différentes filières préalablement autorisées.

\subsection{Application de ces principes}

Les principes évoqués en 1.1. vont permettre aux exploitants du nucléaire de mettre en cuvre sur leur site la gestion correspondante des déchets TFA, selon les règles suivantes ( $c f$. tab. II).

\subsubsection{Le zonage des installations}

Il s'agit de déterminer pour chaque installation, INB ou ICPE, deux types de locaux industriels :

- ceux qui ne peuvent pas produire (de par leur fonction ou celle des matériels qu'ils contiennent) de déchets à radioactivité artificiellement ajoutée et qui seront rattachés à la Zone Déchets Conventionnelle (ex. : station de pompage). À noter qu'en cas «d'incident» éventuel de contamination dans ces locaux, ceux-ci seraient temporairement classés en Zone Déchets Nucléaire en attendant leur réhabilitation et un reclassement en Zone Déchets Conventionnelle;

(*) INB : Installation nucléaire de base

ICPE : Installation classée pour la protection de l'environnement.

(**) CNPE : Centre nucléaire de production d'électricité. 
TABLEAU II

Gestion des déchets issus d'INB - Schéma de principe.

Management of the wastes of nuclear installations: theoretical scheme.

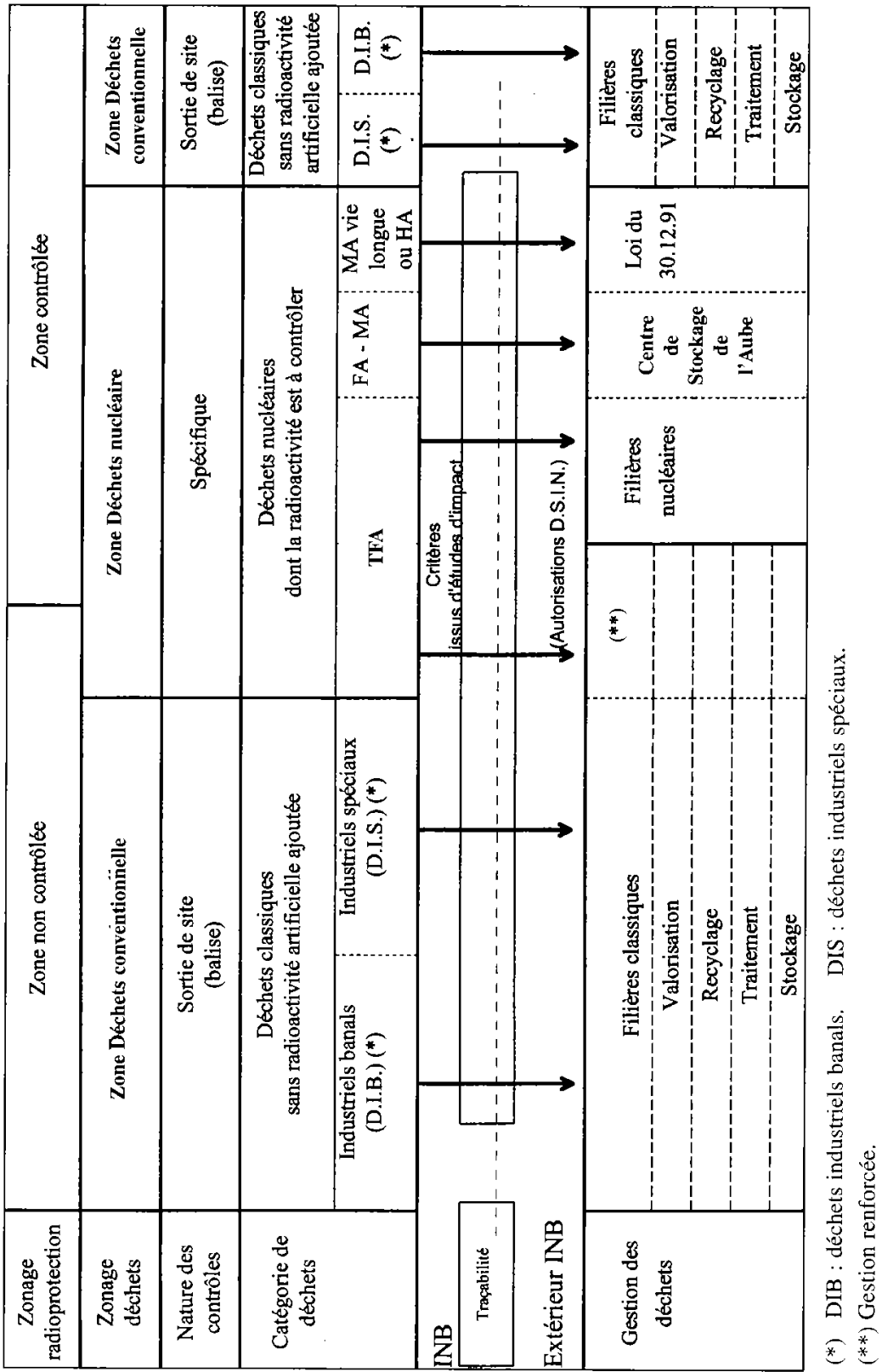


- les autres locaux, susceptibles de produire des déchets à radioactivité artificiellement ajoutée, classés en Zone Déchets Nucléaire. Certains de ces locaux peuvent changer de classe après démantèlement et évacuation des matériels irradiants et contaminés qu'ils contiennent, puis éventuellement assainissement de leurs parois en béton. Tous les déchets qui en sont issus ne sont pas forcément radioactifs (ex.: emballages de pièces neuves introduites dans la zone).

Ce sont les exploitants qui ont la responsabilité de définir ces deux zones et d'en suivre l'évolution, avec information à l'administration.

La distinction de zones permet de trier, grossièrement mais sans risque, les déchets issus d'une installation nucléaire. Les déchets sortant de la Zone Déchets Conventionnelle rejoindront directement un traitement de type classique sans autre contrôle de précaution qu'une mesure globale de type "balise sortie de site ". Les déchets issus de la Zone Déchets Nucléaire rejoindront, soit une filière de traitement/élimination de type nucléaire, soit une filière de type classique à gestion renforcée à condition de satisfaire à des critères précis issus de l'étude d'impact et d'être autorisée par l'administration (cf. § 1.1).

La gestion renforcée applicable aux déchets issus de Zone Déchets Nucléaire, et quel que soit leur devenir (stockage TFA ou recyclage/élimination), mettra en œuvre: - des mesures de radioactivité prouvant que l'on respecte les limites données par l'étude d'impact,

- une traçabilité adaptée des déchets.

\subsubsection{La mise en place de filières de traitement/élimination}

L'étude déchets citée plus haut décrira, pour chaque type de déchet, la gestion retenue et, entre autres, les filières de stockage, traitement, revalorisation, élimination, en fonction des caractéristiques physico-chimiques des déchets, de leur radioactivité et de leur quantité. Le principal intérêt et la nouveauté de ces dispositions viendraient de ce qu'une filière de traitement de type classique à gestion renforcée agréée par les autorités puisse accueillir des déchets issus de la "Zone Déchets Nucléaire» pour peu que ceux-ci satisfassent aux conditions issues d'une étude d'impact. En effet, pourquoi ne pas imaginer que les gravats du démantèlement servent aux soubassements de route ou à des remblais ? Ceci est certainement envisageable pour peu que les quantités, les niveaux d'activité résiduels, les types de réutilisation envisagés, les outils industriels existants ou à créer, soient sans aucun danger vis-à-vis de la santé du public : tous ces critères sont à intégrer dans l'étude d'impact et être satisfaits par les déchets concernés.

À ce titre, quelques filières de valorisation sont en cours d'étude à EDF mais, ces études n'ont pas encore abouti. Celle dont l'avancement est le plus significatif concerne l'incinération avec valorisation énergétique d'huiles TFA. Il est question de cette filière au chapitre 3 . 


\subsubsection{La traçabilité des déchets}

Bien entendu, la mise en place d'études déchets et des filières de traitement/élimination associées doit s'accompagner de la définition de la traçabilité des déchets ainsi expédiés. En effet, il importe de pouvoir retrouver l'origine des déchets chez l'industriel transformeur ou chez le stockeur pour pouvoir satisfaire au principe de responsabilité du producteur. Cette traçabilité consiste en un suivi gestionnaire de l'origine, des quantités, des caractéristiques et du devenir final des déchets produits. Elle permet de suivre le devenir des déchets jusqu'à leur lieu de stockage ou jusqu'à celui de leur transformation en cas de réutilisation.

Dans le cas d'une transformation, on pense que la non nocivité des produits obtenus est la conséquence directe du respect des critères de l'étude d'impact et ce, indépendamment de cette traçabilité : ce point reste à approfondir et sera nécessairement soumis à autorisation administrative du fait que, comme on l'a déjà dit, c'est à l'administration qu'il appartient d'autoriser ou non telle ou telle filière.

\subsubsection{La réalisation des contrôles associés}

La gestion sur sites de ces déchets doit s'accompagner d'une mise en place des contrôles appropriés. C'est ainsi que pour les déchets issus de la «Zone Déchets Conventionnelle», une mesure globale est à réaliser, en sortie de site, au moyen de balises.

Un contrôle, sous la forme d'une mesure de radioactivité, est à réaliser sur site systématiquement pour les déchets issus de la «Zone Déchets Nucléaire» qui seraient évacués dans le cadre de filières afin de s'assurer que ceux-ci vérifient bien les critères retenus pour la filière considérée (respect notamment des activités massiques maximales et des spectres de radionucléides associés, retenus dans l'étude d'impact).

\section{Quels sont les TFA à EDF ?}

\subsection{Description qualitative}

- Les déchets proviennent de Zones Déchets Nucléaire définies dans les plans de zonage des différentes INB (dans le cadre des études déchets citées ci-dessus). Leur activité massique moyenne maximale est de l'ordre de $100 \mathrm{~Bq} / \mathrm{g}$. Les tranches prises en compte sont : les 6 tranches UNGG (CHINON A1, A2 et A3 - ST-LAURENT A1 et A2 BUGEY 1) ; la centrale de CHOOZ A ; les 58 tranches REP.

- Les quantités de déchets correspondent à l'exploitation normale des tranches REP (Durée de vie prévisionnelle de 40 ans) et aux deux niveaux successifs de démantèlement ( $c f$. tab. I). Ces déchets sont: 
- des gravats et terres provenant d'opérations d'assainissement du génie civil de locaux et de chemins de circulation;

- des calorifuges et déchets, amiantifères ou non, provenant du décalorifugeage des circuits et matériels soumis à température, joints et tresses amiantifères de robinetterie, etc.;

- éventuellement des résines $\mathrm{APG}\left(^{*}\right)$, si elles n'étaient pas incinérées à CENTRACO;

- des déchets compactables : filtres, vinyles, plastiques, caoutchoucs;

- des déchets métalliques : tuyauteries, réservoirs, échangeurs, robinetteries, matériels tournants;

- des câbles électriques dont l'isolant peut être contaminé superficiellement.

\subsection{Description quantitative}

Le tableau I indique, pour les déchets classés par appartenance, la répartition selon trois phases (exploitation, démantèlement niveau 2 et démantèlement niveau 3 ) des grandes catégories de déchets : TLC, métalliques et gravats. Les principales hypothèses retenues pour l'estimation des tonnages principaux sont résumées ci-après. C'est le retour d'expérience d'exploitation qui permet l'affectation (ou non) des déchets en classe TFA.

\subsubsection{Démantèlement niveau 2}

Déchets métalliques : les matériels concernés sont les suivants, répartis en six grandes familles, du fait de modes de conditionnement potentiellement différents:

- réservoirs et échangeurs faiblement contaminés;

- robinets, pompes faiblement contaminées, matériels des circuits de ventilation, petits matériels de manutention (hors poutres), charpentes (hors gaines);

- tuyaux faiblement contaminés ;

- gaines de ventilation, capteurs, matériels électriques (hors gros câbles électriques), tôles de calorifuge. Il est prévu de compacter les matériels de cette dernière famille; - gros câbles électriques (diamètre supérieure à $15 \mathrm{~mm}$ ).

Gravats : on ne prend en compte que l'assainissement des plafonds, parois verticales et sols des locaux nucléaires.

En l'absence de mesures précises de contamination incrustée dans le génie civil, on formule les hypothèses suivantes pour des épaisseurs à assainir dans les différents locaux à caractère nucléaire : plafonds : $1 \mathrm{~mm}$; parois verticales (voiles, piliers, murs d'enceinte) : $2 \mathrm{~mm}$; sols de locaux contenant des matériels faiblement ou non contaminés : $1 \mathrm{~cm}$; sols de locaux contenant des matériels fortement contaminés : $20 \mathrm{~cm}$; voiles et fonds de piscines du bâtiment combustible : $1 \mathrm{~m}$.

Les tonnages mentionnés sont obtenus en prenant une densité de 1,5 pour les gravats engendrés par les opérations d'assainissement.

$\left(^{*}\right)$ APG : circuit de purge des générateurs de vapeur. 


\subsubsection{Démantèlement niveau 3}

Déchets métalliques: le circuit primaire serait décontaminé. Les parties du circuit primaire stockées en décharge TFA seraient : les boucles primaires; la partie primaire des générateurs de vapeur; le pressuriseur et sa ligne d'expansion; les pompes primaires hors moteur.

À ces matériels on peut rajouter, probablement sans besoin de décontamination: la partie secondaire des générateurs de vapeur; les moteurs de pompes primaires; les équipements du couvercle de cuve; les outillages de levage et de support des internes de cuve.

Gravats : après démantèlement du circuit primaire, un assainissement du génie civil du bâtiment réacteur sera réalisé. Comme pour le démantèlement de niveau 2, on formule les hypothèses suivantes pour les épaisseurs à assainir: plafonds : $1 \mathrm{~mm}$; parois verticales: $2 \mathrm{~mm}$; sols : $1 \mathrm{~cm}$; voiles et fond de piscine bâtiment réacteur: $1 \mathrm{~m}$ (à assainir après démantèlement des peaux de piscines consécutif au démantèlement de la cuve réacteur et de ses constituants).

\subsubsection{Répartition des tonnages dans le temps}

Les tonnages engendrés jusqu'au démantèlement ultime de l'ensemble des centrales nucléaires sont donc estimés, pour les plus importants d'entre eux, à : déchets métalliques : $500000 \mathrm{t}$; gravats et bétons : $250000 \mathrm{t}$; câbles électriques : $18000 \mathrm{t}$.

Pendant les 20 premières années, le tonnage cumulé sera faible (exploitation REP + démantèlement niveau 2 UNGG) : 40000 à $50000 \mathrm{t}$. Après la fin du niveau 3 des UNGG et du niveau 2 des REP (vers 2050), ce niveau se stabilisera vers $350000 \mathrm{t}$ pendant 20 à 25 ans pour atteindre leurs valeurs maximales à la fin du $21^{\mathrm{e}}$ siècle.

\section{Quelle gestion des TFA est-elle envisagée à EDF ?}

\subsection{Le stockage des déchets TFA}

Concernant la majeure partie des déchets issus des «Zones Déchets Nucléaire » dont le niveau de radioactivité ne permettra pas de les stocker dans une décharge classique et qui ne feront pas l'objet d'une filière de réutilisation telle que vue dans le paragraphe 1.2.2, il convient de prévoir un lieu de stockage ad-hoc, car SOULAINES, stockage de surface FMA, est limité en capacité et économiquement inadapté aux déchets de cette catégorie. 
Une première étude visant à caractériser un tel centre de stockage a été menée avec l'ANDRA et a montré qu'un tel stockage pouvait accueillir ces déchets en vrac, c'est-à-dire sans conditionnement spécifique autre que celui relatif aux caractéristiques physico-chimiques du déchet, et ce sans nécessiter de caractéristiques géologiques particulières du terrain hôte (sous-sol stable et de perméabilité limitée). Son dimensionnement tient compte des productions des autres industriels, CEA et COGEMA, notamment. La surveillance d'un tel centre de stockage ne devrait pas s'exercer bien au-delà de la simple durée d'exploitation du centre, tout au plus quelques années.

L'ANDRA et France-Déchets ont proposé, dans un avant-projet sommaire commun, une infrastructure du site permettant, selon la nature des déchets, leur stockage soit par alvéole, soit en vrac, soit conteneurisés. Certaines ferrailles peuvent subir avant stockage, un traitement de type cisaillage/compactage afin d'optimiser l'utilisation du vide de fouille et la résistance mécanique à l'affaissement.

\subsection{La mise en place de filières de traitement/élimination}

Quelques filières de valorisation/élimination sont en cours d'étude à EDF. La filière dont l'étude est la plus avancée concerne l'incinération avec valorisation énergétique d'huile faiblement contaminée. Cette étude a été présentée à la DSIN et pourrait constituer une première réutilisation dans l'industrie classique (avec gestion renforcée) de déchets TFA. Rappelons que l'étude d'une filière se doit de justifier tous les éléments de la chaîne de gestion des déchets, de sa production jusqu'à sa destination finale.

\subsubsection{Origine, quantités entreposées et caractérisation des huiles TFA d'EDF}

À ce jour, un peu plus de $1000 \mathrm{~m}^{3}$ de telles huiles sont répartis et entreposés sur les différents sites de production. Ces huiles proviennent, soit de vidanges de matériels tournants en exploitation, soit de centrales en arrêt définitif de production. La production annuelle représente environ $135 \mathrm{~m}^{3}$ pour l'ensemble des sites en exploitation. $90 \%$ de ces huiles ont une activité totale moyenne inférieure à $5 \mathrm{~Bq} / \mathrm{g} ; 75 \%$ inférieur à $1 \mathrm{~Bq} / \mathrm{g}$ et $65 \%$ inférieur à $0,5 \mathrm{~Bq} / \mathrm{g}$. Les radio-nucléides contenus dans ces huiles sont par ordre décroissant d'importance : ${ }^{60} \mathrm{Co}: 70 \%$ en moyenne de la radioactivité totale ; ${ }^{110} \mathrm{Ag}: 15 \% ;{ }^{137} \mathrm{Cs}: 8 \% ;{ }^{58} \mathrm{Co}: 4 \% ;{ }^{134} \mathrm{Cs}: 1,5 \%$.

\subsubsection{Les différentes filières huiles TFA étudiées}

L'incinération dans un incinérateur de déchets industriels : l'huile TFA, mélangée ou non à d'autres huiles, servirait de combustible pour incinérer d'autres déchets ( 1 tonne d'huile pour 12 à 13 tonnes de déchets). Les mâchefers produits, représentant 10 à $15 \%$ du poids des produits entrants, seraient expédiés vers un centre d'enfouissement technique de classe 1 . 
L'incinération en cimenterie: 1'huile TFA serait injectée au niveau des brûleurs, les cendres produites étant recyclées en tête de four et se retrouveraient dans la poudre de ciment.

Le recyclage: l'huile serait centrifugée et utilisée sur les chantiers de travaux publics pour faciliter le démoulage de structures en béton. Les résidus de centrifugation pourraient être incinérés.

\subsubsection{Les études d'impact associées}

Le but de l'étude d'impact est de déterminer la valeur de l'activité maximale moyenne et le spectre enveloppe des radio-nucléides contenus dans les huiles, pour lesquels l'impact radiologique (dose individuelle reçue par les travailleurs acteurs de la filière et dose collective des groupes de population concernés par chaque étape du processus) peut être considéré comme acceptable. Pour réaliser les calculs nécessaires à l'étude d'impact, les hypothèses suivantes ont été retenues :

Hypothèses générales : le stock de $1000 \mathrm{~m}^{3}$ d'huile est scindé en 4 classes d'activité, de valeurs maximales: 0,$5 ; 1 ; 5$ et $10 \mathrm{~Bq} / \mathrm{g}$.

En général, les quatre séries de calculs d’impact (un par classe) ont été effectués.

Pour chaque classe d'activité, les calculs sont fondés sur un spectre enveloppe correspondant, pour chaque radio-isotope considéré, à la valeur maximale constatée sur l'ensemble des sites, ce qui est par ailleurs pénalisant.

Hypothèses concernant le transport : on a supposé que les opérations de collecte (transport, dépotage et entretien des matériels) étaient exécutées par le même employé ce qui est également pénalisant. L'évaluation de l'impact sur le conducteur a été réalisée dans trois cas : citernes de 10 et $30 \mathrm{~m}^{3}$ d'huiles contaminées ainsi que $1^{\text {re }}$ alvéole de la citerne derrière le chauffeur remplie de fluide non contaminé et constituant un écran biologique (transport mixte). On a considéré le cas d'une collecte régionale, déjà existante pour des huiles ordinaires, réalisée à la vitesse moyenne de $50 \mathrm{~km} / \mathrm{h}$.

Hypothèses concernant les filières : pour chaque fillière, deux scénarios ont été étudiés:

- par lots : l'huile contaminée sert de combustible pour brûler d'autres déchets ; - par année : l'huile contaminée est mélangée à d'autres combustibles.

Les mâchefers issus de l'incinération de tout le stock ont été considérés comme transportés par le même employé, hypothèse de nouveau pénalisante. L'impact sur les employés du CET n'a pas été étudié, mais il est, en première approximation, comparable à l'impact obtenu dans le cas du scénario de «l'oubli immédiat » après la fin d'exploitation (cas de la résidence bâtie sur le CET). 


\section{Principaux résultats:}

- la personne la plus exposée dans le cadre de la collecte serait soumise à une dose inférieure à $10 \mu \mathrm{Sv} /$ an pour un transport mixte (classe $1 \mathrm{~Bq} / \mathrm{g}$ ) ou comprise entre 10 et $100 \mu \mathrm{Sv} /$ an pour tout transport (classe $5 \mathrm{~Bq} / \mathrm{g}$ ).

Dans le cas de l'incinération, c'est le transporteur des mâchefers entre l'incinérateur et le CET classe 1 qui serait le plus exposé, à raison d'une dose de $10 \mu \mathrm{Sv} / \mathrm{an}$ (classe $5 \mathrm{~Bq} / \mathrm{g}$ ) ou $3 \mu \mathrm{Sv} /$ an (classe $1 \mathrm{~Bq} / \mathrm{g}$ );

- la construction d'immeuble avec un béton provenant d'un four de cimenterie induira la dose individuelle la plus forte pour le maçon; les doses reçues seraient de $7 \mu \mathrm{Sv} / \mathrm{an}$ (classe $5 \mathrm{~Bq} / \mathrm{g}$ ) ou de $2 \mu \mathrm{Sv} / \mathrm{an}$ (classe $1 \mathrm{~Bq} / \mathrm{g}$ );

- enfin, deux étapes du recyclage conduiraient aux doses suivantes:

- manipulation des déchets de procédé : 14 et $4 \mu \mathrm{Sv} / \mathrm{an}$ (classe 5 et $1 \mathrm{~Bq} / \mathrm{g}$ );

- rémanence de l'activité dans le béton décoffré : 16 et $5 \mu \mathrm{Sv} / \mathrm{an}$ (classe 5 et $1 \mathrm{~Bq} / \mathrm{g})$.

À titre de comparaison, rappelons que l'exposition naturelle en France est de $2400 \mu \mathrm{Sv} / \mathrm{an}$ et que celle due aux rejets des centrales nucléaires est estimée à $1 \mu \mathrm{Sv} / \mathrm{an}$.

\subsubsection{Le choix de la filière huiles TFA}

L'impact radiologique du transport serait le même, quelle que soit la filière envisagée. Ce n'est donc pas un éléments déterminant du choix, même si son incidence est prépondérante dans l'impact global ( 5 à 10 fois supérieur aux impacts des traitements des trois filières respectives : incinération-cimenterie-recyclage).

L'incinération et l'élimination en cimenterie présenterait un même impact au niveau individuel, mais deux fois plus faible que celui dû au recyclage. Au niveau collectif, les impacts dus respectivement à la cimenterie et au recyclage seraient dix fois plus élevés que celui dû à l'incinération (populations concernées plus importantes). De plus, l'incinération permettrait de détruire les huiles tout en maîtrisant les rejets et les déchets engendrés sans remettre des produits en circulation dans le domaine «dit public » contrairement aux autres filières envisagées. L'incinération garantirait, en l'occurrence, une meilleure traçabilité.

Toutes les raisons évoquées ci-dessus font qu'EDF priviligierait la filière «incinération », dont le schéma général est joint dans la figure 1.

\subsubsection{Mise en auvre de la filière huiles TFA}

L'incinérateur appartiendrait à une ICPE dont il conviendra de clarifier la situation administrative. La description et les spécifications de la filière seraient à mettre au point entre le (ou les) industriel (s) retenu (s) et EDF et à agréer par la DSIN. Un certain niveau d'assurance qualité devrait garantir la traçabilité des huiles jusqu'à leur 


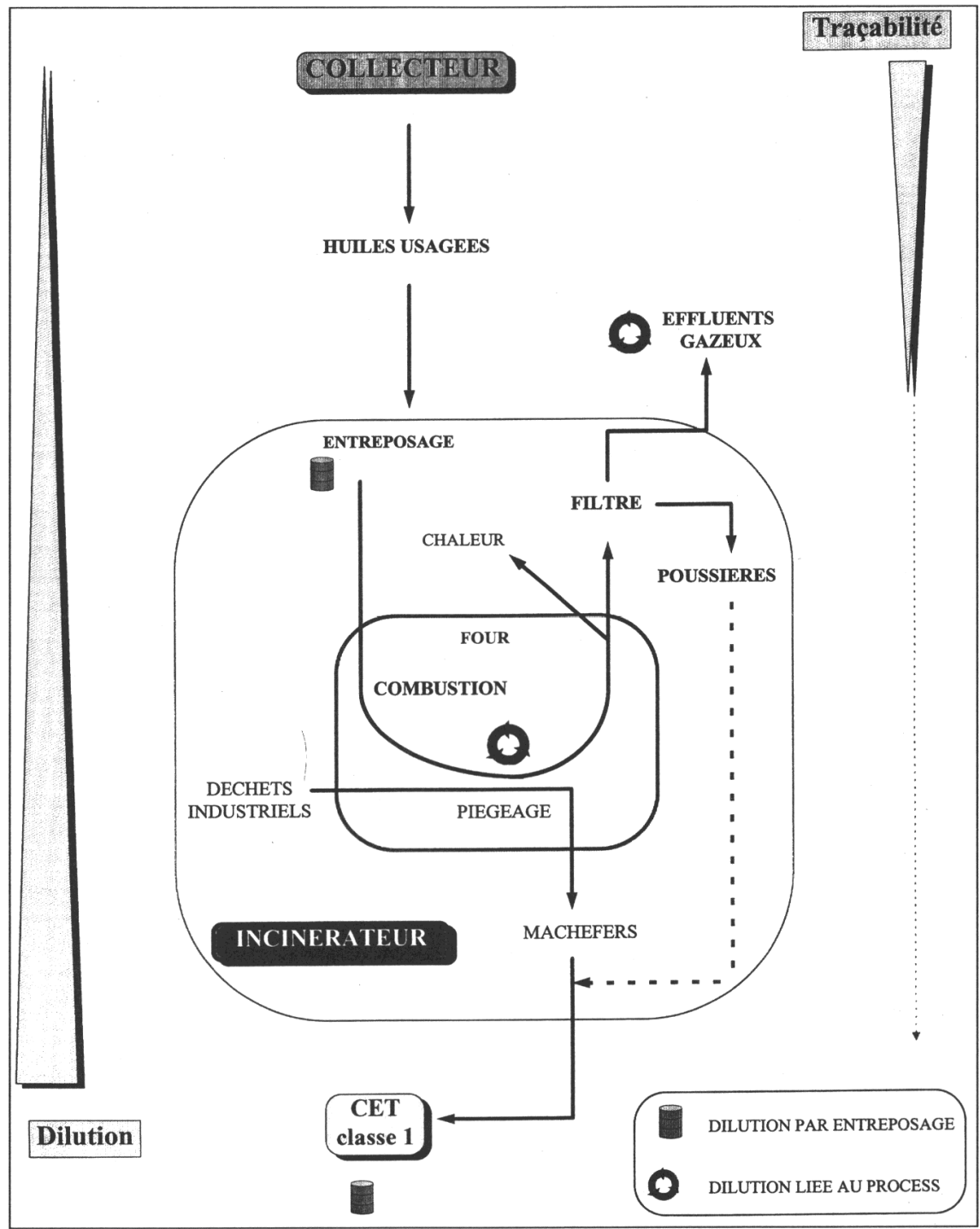

Fig. 1. - Élimination en incinérateur de déchets industriels.

traitement chez l'incinérateur. Une difficulté, et non des moindres, consiste à trouver un industriel qui accepte de prendre les huiles TFA dans son installation. Les réticences apparaissent clairement à ce niveau. De ce point de vue, la mise en œuvre de cette filière constituerait un prototype. 


\subsubsection{Autres études en cours}

L'IPSN a réalisé l'étude d'impact du processus de vitrification par torche à plasma des déchets amiantifères TFA. Les vitrifiats sont supposés être stockés en CET classe 1 ou utilisés en soubassement de route. Enfin, une filière relative aux ferrailles légèrement contaminées ou activées que l'on destine à la future décharge TFA fait également partie intégrante des études filière TFA (à noter que la fusion à SOCODEI devrait accueillir les ferrailles FMA ainsi que celles issus du démantèlement UNGG et devrait servir à la fabrication des protections biologiques des colis irradiants destinés à SOULAINES).

\section{Conclusion}

La gestion des déchets TFA est en transition entre le stade de la réflexion et celui de la mise en œuvre, notamment sur les aspects zonage des installations, études déchets et stockage TFA. Ce tournant important concerne principalement les aspects déchets issus de démantèlement bien plus que ceux issus de l'exploitation.

Les démantèlements de Brennilis, Chinon A, St-Laurent A, Bugey 1 et Marcoule ont commencé parallèlement à ces réflexions : leur organisation s'efforce de prendre en compte ces nouvelles orientations en les adaptant aux processus en cours ainsi qu'à la mise en service prochaine des installations d'incinération et de fusion de CENTRACO.

S'il est vrai que ces déchets ont été la cible des écologistes ces dernières années, on peut noter que les nombreuses communications publiques sur ce sujet, faites à partir de 1995 (Entretiens de Ségur, Assises de la Baule, journées SFEN, auditions et rapport Birraux) ont permis de calmer les esprits et de constater que les orientations et concepts/projets proposés n'ont pas déclenché de vives critiques. 